\title{
Full sky study of diffuse Galactic emission at decimeter wavelenghts ${ }^{\star}$
}

\author{
P. Platania ${ }^{1}$, C. Burigana ${ }^{2}$, D. Maino ${ }^{1}$, E. Caserini ${ }^{1}$, M. Bersanelli ${ }^{1,3}$, B. Cappellini $^{1}$, and A. Mennella ${ }^{3}$ \\ 1 Dipartimento di Fisica, Università degli Studi di Milano, via Celoria 16, 20133 Milano, Italy \\ 2 IASF/CNR, Sezione di Bologna, via P. Gobetti 101, 40129 Bologna, Italy \\ 3 IASF/CNR, Sezione di Milano, via Bassini 15, 20133 Milano, Italy
}

Received 5 March 2003 / Accepted 24 July 2003

\begin{abstract}
A detailed knowledge of the Galactic radio continuum is of high interest for studies of the dynamics and structure of the Galaxy as well as for the problem of foreground removal in Cosmic Microwave Background measurements. In this work we present a full-sky study of the diffuse Galactic emission at frequencies of few GHz, where synchrotron radiation is by far the dominant component. We perform a detailed combined analysis of the extended surveys at 408, 1420 and $2326 \mathrm{MHz}$ (by Haslam et al. 1982; Reich 1982; Reich \& Reich 1986; Jonas et al. 1998, respectively). Using the technique applied by Schlegel et al. (1998) to the IRAS data, we produce destriped versions of the three maps. This allows us to construct a nearly-full-sky map of the spectral index and of the normalization factor with sub-degree angular resolution. The resulting distribution of the spectral indices has an average of $\bar{\beta}=2.695$ and dispersion $\sigma_{\beta}=0.120$. This is representative for the Galactic diffuse synchrotron emission, with only minor effects from free-free emission and point sources. The maps produced in this work are available via ftp at cdsarc.u-strasbg.fr
\end{abstract}

Key words. Galaxy: general - methods: data analysis

\section{Introduction}

The detailed study of radio continuum emission allows a direct evaluation of important parameters describing the dynamics and structure of the Galaxy, such as magnetic field distribution and electron energy distribution. Moreover, diffuse Galactic radio emission is one of the main sources of unwanted signal in observations of the Cosmic Microwave Background (CMB) at $v \lesssim 50 \mathrm{GHz}$. High precision imaging of the CMB fluctuations is a unique cosmological tool to determine fundamental parameters that describe the structure and evolution of the Universe and directly probes the early phase of cosmic history.

The new generation of CMB imaging experiments (in particular the $W M A P^{1}$ and $P L A N C K^{2}$ space missions) call for extended, detailed information of Galactic foreground emission. While such exquisite precision measurements provide a great information on foreground emission at microwave frequencies,

Send offprint requests to: P. Platania,

e-mail: Paola.Platania@mi.infn.it

* The maps produced in this work are only available in electronic form via anonymous ftp to cdsarc.u-strasbg. fr $(130.79 .128 .5)$ or via

http://cdsweb.u-strasbg/cgi-bin/qcat?]/A+A/410/847

Colour figures are only available in the electronic edition of this paper

via http://www. edpsciences.org

${ }^{1}$ http://map.gsfc.nasa.gov/

2 http://astro.estec.esa.nl/Planck/ a deep knowledge at radio and sub-millimetre frequencies of all emissions that are between us and the Last Scattering Surface where $\mathrm{CMB}$ photons originate contribute significantly to the accurate exploitation of microwave data. In particular great attention has to be devoted to Galactic foreground emission with their own power spectra and frequency dependence.

The three main physical processes that produce Galactic diffuse emissions are synchrotron, free-free (or thermal bremsstrahlung) and dust emission. While free-free does not dominate in any observed frequency band, synchrotron and dust are the major contributors at low and high frequencies, respectively. Therefore, it is convenient to deal with these two types of foreground emission using template maps obtained from observations where such emissions overwhelm the others. In this way it is possible to cross-correlate experimental CMB data with external data sets (Banday et al. 2003; Bennett et al. 2003) to clean CMB signal from foreground signal as well as performing component separation with the use of priors on foreground emissions (Maino et al. 2003).

As for the dust emission is concerned, a detailed work has been done by Schlegel et al. (1998, hereafter SFD) to combined IRAS with $C O B E$-DIRBE data in order to obtain a template for thermal dust emission with IRAS angular resolution and COBE-DIRBE calibration quality.

In the case of synchrotron emission, observations suffer from many systematic effects which depend on the specific 
experimental setup and observing strategies. Due to the need of covering the whole sky, many instruments perform observations that will be finally combined in a unique data set. This introduces problems such as non-uniform zero-levels, resulting in a normalization of the signal when combining different patches of observation taken by different instruments located in different places. Furthermore the involved technology makes use of radiometer detectors which typically show $1 / f$ noise induced by gain fluctuations that leads to striping effects in the maps when combined with the observing strategy.

In this paper we address the issue of cleaning low frequency maps from their striping effect employing a technique similar to that used by SFD on the IRAS plates. We apply this to the three largest low frequency maps: the $408 \mathrm{MHz}$ map by Haslam et al. (1982), the $1420 \mathrm{MHz}$ map from Reich (1982) and Reich \& Reich (1986), and the 2326 MHz map by Jonas et al. (1998).

Our goal is to produce reliable templates of spectral index and normalization factor of the power law description of the Galactic emission at $\approx \mathrm{GHz}$ frequencies, by removing the main observation artifacts and taking into account the survey calibration and zero level uncertainties. As the recent WMAP results show, any template of the Galactic emission is frequency dependent (Bennett et al. 2003). A comparison between the synchrotron spectral index map at WMAP frequencies and that derived from the analysis of radio surveys at $\approx \mathrm{GHz}$ frequencies will add information on the physical processes of electron energy loss and the properties of Galactic structures.

This paper is organized as follows. In Sect. 2 we briefly summarize the main properties of the considered surveys. The description of the first steps of the adopted data analysis method, source identification and destriping, is given in Sect. 3, while in Sect. 4 we discuss the minimization of the discontinuities of the maps of spectral index and normalization factor of the radio emission power law due to the survey calibration and zero level errors and present our results. Finally, we discuss the results and draw our main conclusions in Sect. 5.

\section{Data description}

Synchrotron radiation, arising from cosmic ray electrons spiralizing into the interstellar magnetic field, is dominant over bremsstrahlung (free-free) emission up to $10-20 \mathrm{GHz}$. The emission spectrum has a power law shape, whose spectral index varies across the sky reflecting variations of the electrons density and magnetic field; a realistic model of the synchrotron emission has to take into account this significant spatial changing, besides the steepening of the spectrum with increasing frequency, due to the increase of energy losses by high energy electrons. Free-free emission comes from electron-ion scattering with a power law shape in frequency; since the diffuse emission at $\approx \mathrm{GHz}$ frequency is dominated by synchrotron radiation, no free-free templates are available at this frequencies.

The only full sky map at decimeter range is the well known $408 \mathrm{MHz}$ map (Haslam et al. 1982). Obtained from four different experiments, the overall map has an angular resolution of $0.85^{\circ}$ and a full beam brightness temperature of $2 \mathrm{~K}$. The estimate of calibration and zero level errors quoted by the authors are $10 \%$ and $3 \mathrm{~K}$ respectively. The $1420 \mathrm{MHz}$ map (Reich 1982; Reich \& Reich 1986), covering the sky at $-19^{\circ}<\delta<90^{\circ}$, has a quoted full beam brightness temperature of $50 \mathrm{mK}\left(0.59^{\circ}\right.$ angular resolution). The authors estimate $5 \%$ of calibration error and $0.5 \mathrm{~K}$ error of the zero level.

The higher frequency map (2326 MHz, Jonas et al. 1998), covering the sky region $-83^{\circ}<\delta<13^{\circ}$ for $0 \mathrm{~h}<\mathrm{RA}<12 \mathrm{~h}$ and $-83^{\circ}<\delta<32^{\circ}$ for $12 \mathrm{~h}<\mathrm{RA}<24 \mathrm{~h}$, has an angular resolution of $19^{\prime}$ and a full beam brightness temperature of $25 \mathrm{mK}$. Calibration and zero level errors are 5\% and $80 \mathrm{mK}$, respectively. The receiver was sensitive to linearly polarized radiation.

The combination of gain drifts and observational strategy results in artificial structures in the three final maps: these appear like stripes particularly intense in the scan directions. A careful analysis of the systematic effects in the 408 and $1420 \mathrm{MHz}$ maps has been performed by Davies et al. (1996). Following their considerations, the most evident artifacts in the $408 \mathrm{MHz}$ map are the nearly vertical stripes at constant right ascension generated by the elevation scanning strategy and not completely eliminated by the nodding scan technique used in the experiment. Horizontal stripes are also evident at declination $<20^{\circ}$. In the case of the $1420 \mathrm{MHz}$ map the inclined striations reflect the azimuthal scanning strategy. Further artifacts show up in the horizontal direction mostly in the declination range between $20^{\circ}$ to $50^{\circ}$. After point source extraction, the authors remove the major Fourier components which produce horizontal and vertical striations on angular scales $<5^{\circ}$ in the Haslam map and diagonal and horizontal striations on scales $<5^{\circ}$ and $<9^{\circ}$ respectively in the $1420 \mathrm{MHz}$ map. As they point out, this technique removes the easily visible angular frequency leaving spurious structures on scales of several tens of degrees. Vertical striations parallel to the scanning direction are visible in the $2326 \mathrm{MHz}$ map too and remain even after the smoothing applied by Jonas et al. (1998).

\section{Description of the destriping method}

To clean the data from the artifacts mentioned above, it is necessary to select the contaminated directions and remove the excess signal; for this purpose we adapted the method proposed by SFD to destripe the IRAS map. We remember that, although a deep comprehension of the experimental/observational origin of stripes in the considered survey certainly helps the data exploitation, this and other kinds of destriping algorithms (see e.g. Keihänen al. 2003, and references therein, for a different destriping approach) have the remarkable property to work almost independently from the details of the stripe pattern. For a comprehensive explanation of the algorithm concept we refer to their original paper; here we explain briefly the main steps of the process, that are also summarized in the block diagram of Fig. 1. The analysis is not performed on the map as a whole, since the code works on "squared" patches. We divided the maps of the surveys at 408, 1420, and $2326 \mathrm{MHz}$ respectively into 44,33 , and 22 partially overlapping patches of $152 \times 152$ pixels with angular size $\Delta \theta \times \Delta \phi=0.35^{\circ} \times 0.35^{\circ}$. The destriping code acts on maps gridded in standard polar coordinates $\theta, \phi$ by using an Equi-Cylindrical Projection (ECP) 


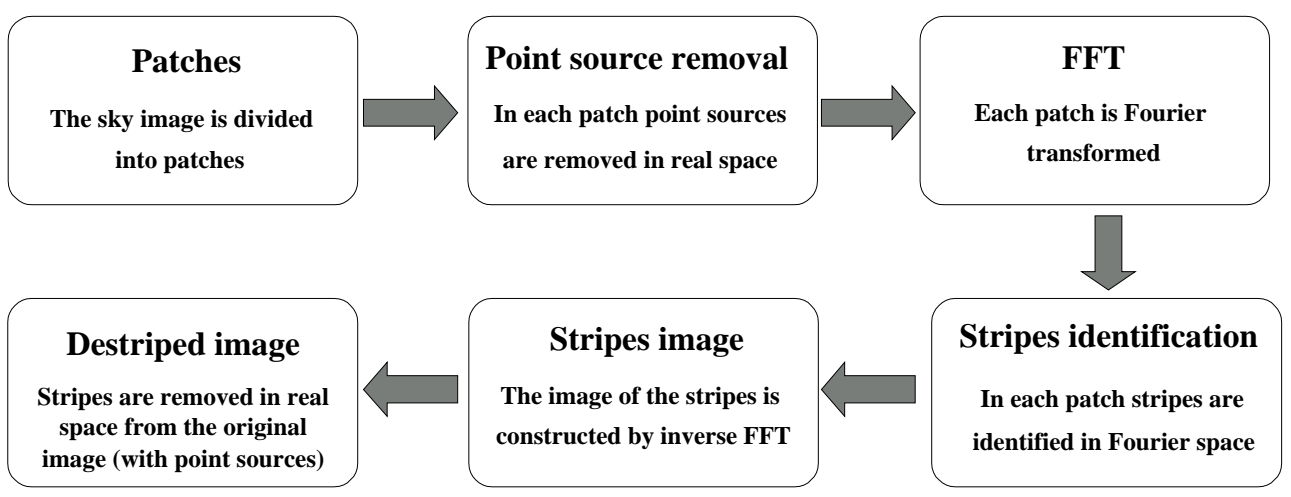

Fig. 1. The block-diagram summarizes the steps of the destriping procedure.
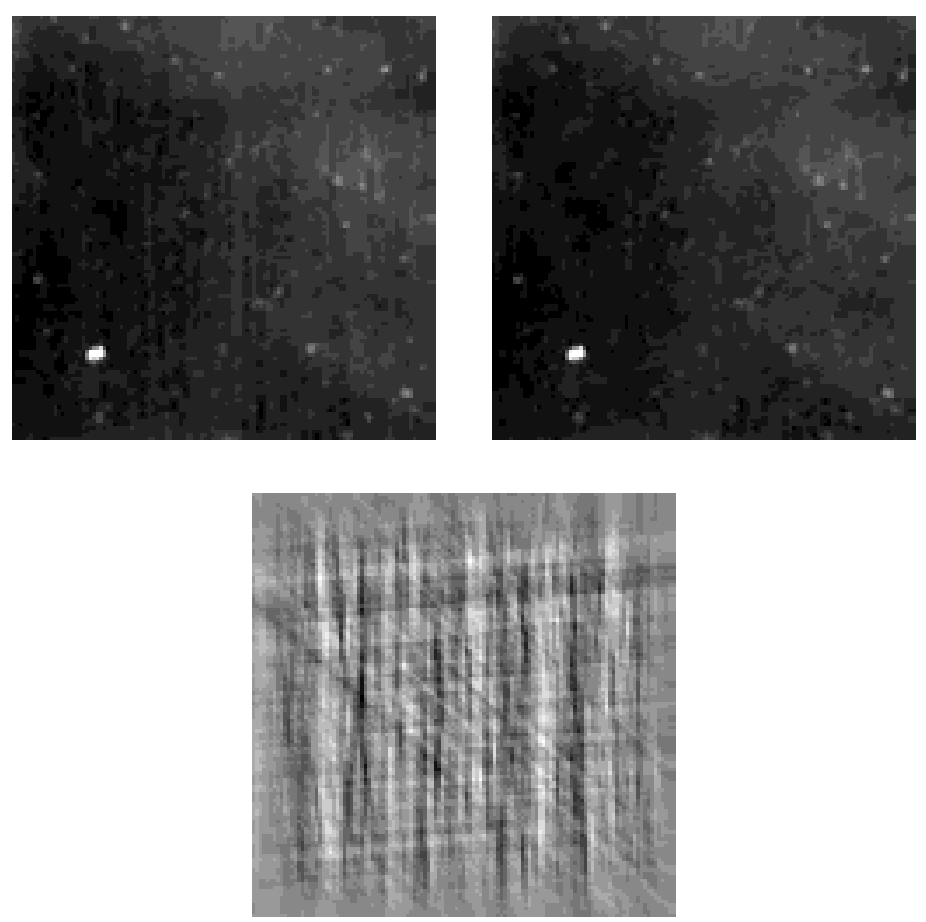

Fig. 2. A patch of the $408 \mathrm{MHz}$ map is shown (left) together with the same destriped (right). The lower is the stripe image; note the diagonal features together with the dominant vertical striations.

pixelization, i.e. a rectangular, not equal area, projection of the sphere.

The algorithm first performs a point source removal, selecting regions whose signal exceeds the median filtered image by a given threshold. The source signal is then replaced with the median signal from an anulus around the source (SFD); this allows destriping to be performed in the Fourier space, where power from bright sources may add and overwhelm many wavenumbers.

The Fourier domain, parameterized in polar coordinates $\left(k_{r}\right.$, $k_{\theta}$ ), is then divided into 90 bins of $2^{\circ}$ in $k_{\theta}$ and a background power $P_{\text {back }}$ is calculated by "finding the median at each $\theta$ and then median filtering the result" (SFD). The contaminated wavenumbers are those for which the power ratio $\gamma_{\theta}$ between the power, $P_{\theta}$, in each $k_{\theta}$ bin and the background power, $P_{\text {back }}$, differs significantly from unity. The difference, $\Delta P_{\theta}$, between the power as a function of $\theta$ and a mean "good" power, $P_{\text {ave }}$ (obtained by averaging all power at each $\left(k_{\mathrm{r}}, k_{\theta}\right)$ with $\gamma_{\theta}$ lower than the stripes threshold $\bar{\gamma}$ ), is then inversely transformed to get the image of stripes only, to be subtracted from the original patch.

The original resolution of the $2.326 \mathrm{GHz}$ map is approximately the same as the pixel size used in this analysis; we tested the influence of a better sampling on the destriping procedure adapting the algorithm to the same map with $2048 \times$ 1024 pixels (each pixel of $0.175^{\circ} \times 0.175^{\circ}$ ). The results show that the pixel by pixel temperature difference between the same destriped patches with different pixel size is, on average, less than $5 \%$ for $70-80 \%$ of the pixels. Given that the three frequencies maps will be jointly used in the following analysis, we choosed to perform the destriping on the $2.326 \mathrm{GHz}$ map with the same pixel size as the others.

The real space image of a typical Haslam patch is shown in Fig. 2 together with the same patch after destriping. In the same figure the real space image of the subtracted stripes is 


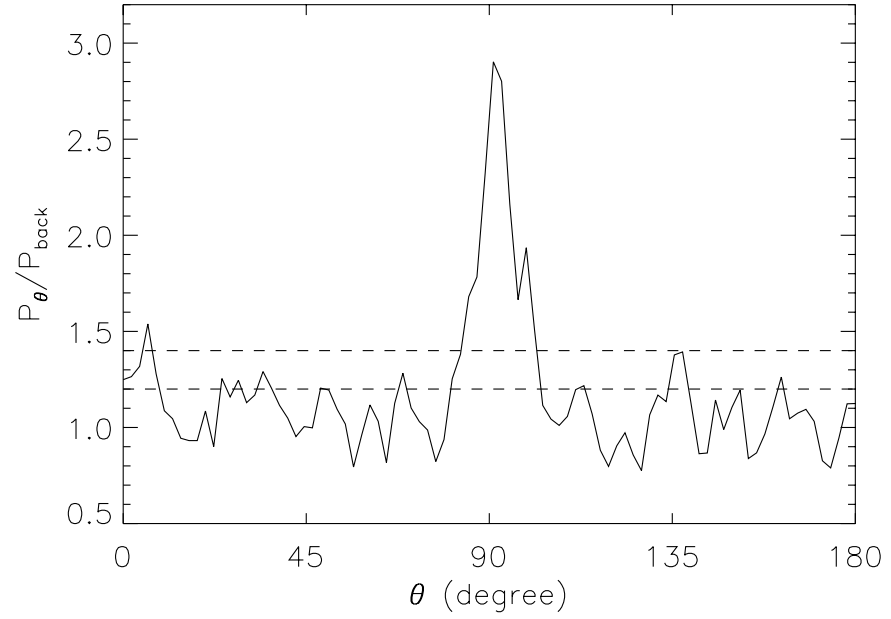

Fig. 3. Ratio between the power, $P_{\theta}$, in each $k_{\theta}$ bin and the background power, $P_{\text {back }}$, for the same patch of Fig. 2 as a function of the angle $\theta$. Different thresholds $\bar{\gamma}$ are shown (horizontal dashed lines). The highest value selects only very intense stripes; by lowering the threshold, lower peaks are also included.

shown. The destriped patches are then joined together to obtain the destriped map ${ }^{3}$.

\subsection{Determination of the thresholds}

Criteria for the choice of the source extraction threshold $h_{\mathrm{s}}$ and the stripes threshold $\bar{\gamma}$ have to be carefully addressed in order to optimize the destriping procedure. We performed several tests to determine the best thresholds for source and stripe removal and to estimate the relative errors and the effect on the destriped maps. The choice of the thresholds does not have the same effect on every patch of one map. However, the values of $h_{\mathrm{s}}$ and $\bar{\gamma}$ have been mantained fixed for all the patches of the same map, since the division of each map in patches is useful in the analysis but does not reflect a real experimental situation. We therefore look for the values that give the most uniform results on the whole map.

A first test for the effectiveness of the source removal procedure is to look at the cleaned image to check for the presence of residual sources. It should be noted that the optimal choice for stripe threshold $\bar{\gamma}$ is strictly related to source removal. Reminding that the goal is to obtain an image of stripes to be subtracted to the original patch, two opposite non-optimal choices of the source threshold $h_{\mathrm{s}}$ could affect the destriping method. The choice of a high threshold $h_{\mathrm{s}}$ (at limit, avoid source subtraction at all) implies that power from unsubtracted sources could be attributed to stripes and then uncorrectly removed from the original map. On the other hand, the choice of a too low threshold $h_{\mathrm{s}}$ implies that not only the signal from point sources is subtracted but also power from stripes that will not be removed in the destriping procedure.

To optimize the stripe removal, in the Fourier space we first eliminate the peaks higher than $\bar{\gamma}$ as a function of the

\footnotetext{
3 For three patches of the Haslam map the procedure failed in finding a lower signal anulus to replace the source signal; in these cases the algorithm did not perform destriping.
}

Table 1. Distribution of percentage temperature variation $\delta T$ values in four different ranges and the corresponding pixel percentage. $\delta T$ is evaluated as the difference after and before destriping.

\begin{tabular}{c|cccc}
\hline \hline & $\delta T<1 \%$ & $1 \%<\delta T<5 \%$ & $5 \%<\delta T<10 \%$ & $\delta T>10 \%$ \\
\hline 408 & $36.4 \%$ & $55 \%$ & $7.0 \%$ & $0.6 \%$ \\
1420 & $89.14 \%$ & $10.7 \%$ & $0.15 \%$ & $0.01 \%$ \\
2326 & $18.3 \%$ & $50.6 \%$ & $22.5 \%$ & $8.6 \%$ \\
\hline
\end{tabular}

direction $k_{\theta}$ (see Fig. 3). Stripes have a high level of directionality and this gives a good criterion for the choice of the threshold value: $\bar{\gamma}$ has to be not too low in order to maintain this feature and avoid subtraction of real astrophysical signals. On the other side, a too high $\bar{\gamma}$ would eliminate only stripes with very high signal, often in one single direction. Figure 3 shows the pattern of the ratio $P_{\theta} / P_{\text {back }}$ as a function of the angle $\theta$ for the same patch of Fig. 2.

After the identification of the optimal stripe threshold $\bar{\gamma}$, we tested the influence of this choice on the destriped map, comparing it with the same map destriped with a higher value of $\bar{\gamma}$. By changing $\bar{\gamma}$ by $20 \%$ (i.e. to a value well beyond a reasonable choice of the threshold), the number of pixels for which the temperature changes more than $10 \%$ are less than $\simeq 1 \%$ in the $408 \mathrm{MHz}$ map, $\simeq 0.1 \%$ at $1420 \mathrm{MHz}$, and reaches a few percent in the $2326 \mathrm{MHz}$ map (each fraction is referred to the sky coverage of each survey). As shown in Table 1 the destriping procedure does not change the intensity level of the surveys except for a small percentage of the pixels for the 408 and $1420 \mathrm{MHz}$ maps and for less than $10 \%$ of the pixels for the $2326 \mathrm{MHz}$ map.

\subsection{Resulting maps}

The resulting maps are shown in Figs. 4-6. We also produced the three destriped maps without point sources using the same technique that has been implemented in the destriping algorithm. The technique works well for isolated sources; in the case of extended structures, however, the replacement of the high source signal with the median background cause the smoothing of the structure borders. We thus preferred to leave the sources in the maps; their contribution to the spectral index distribution will be discussed in Sect. 4.3. Maps are also available in HEALPix ${ }^{4}$ format (Gòrski et al. 1999) with $N_{\text {side }}=256$. The conversion from ECP to HEALPix maps is performed working in real space via an intermediate step, i.e. an HEALPix map with higher resolution $\left(N_{\text {side }}=4096\right.$, the maximum available within HEALPix packages). For each HEALPix pixel of the higher resolution map, the conversion code $^{5}$ finds the corresponding ECP pixel and assign its signal value. The map is finally degraded to $N_{\text {side }}=256$.

\footnotetext{
4 http://www . eso.org/science/healpix

5 Details on the conversion procedure can be found in Cappellini et al. (2003a,b).
} 

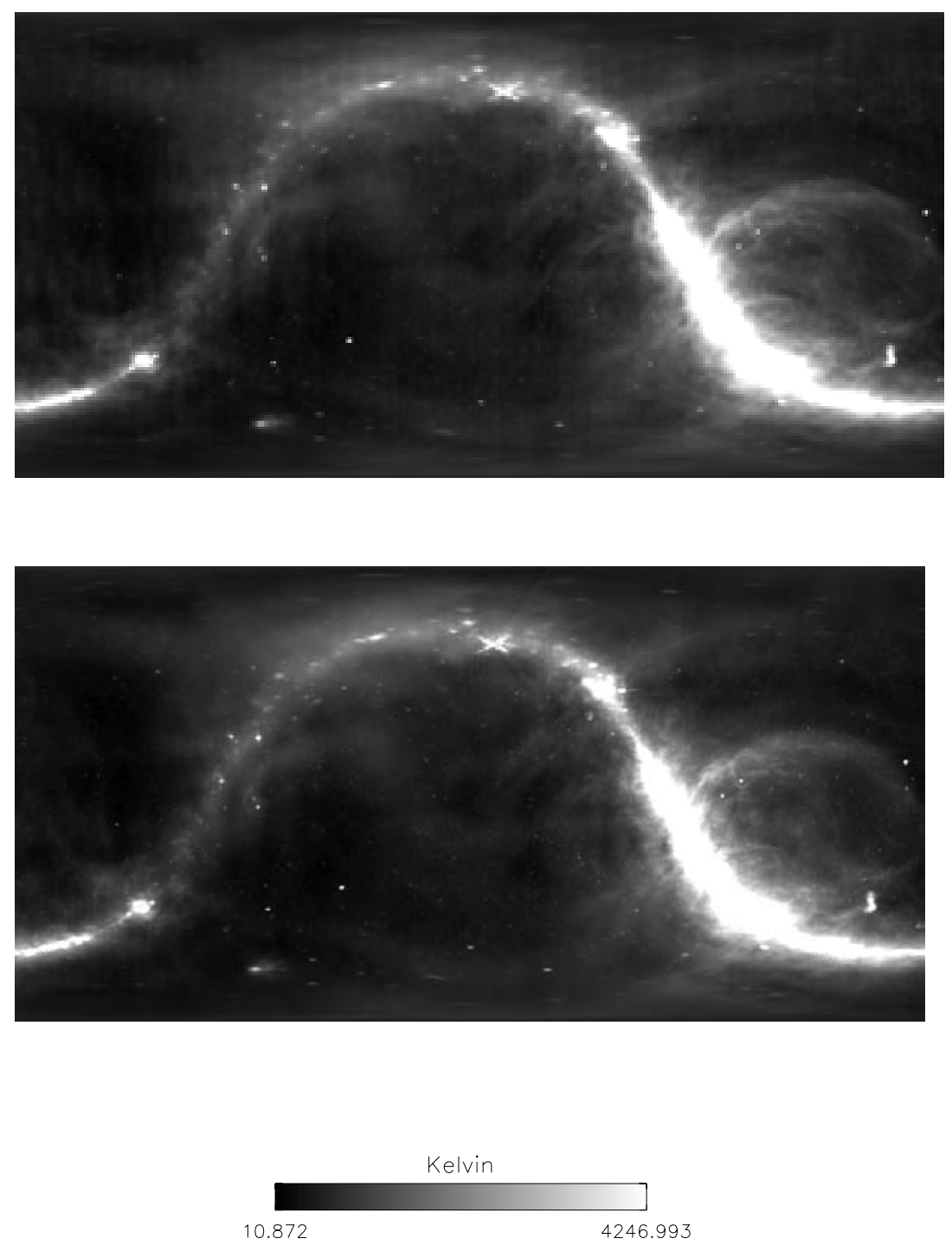

Fig. 4. Top: original $408 \mathrm{MHz}$ map (Haslam et al. 1982). Bottom: destriped $408 \mathrm{MHz}$ map (this work).

\section{Spectral index and normalization factor maps}

One of the main goals of this work is the study of the spectral index of the Galactic diffuse emission over the whole sky, except the region $-90^{\circ}<\delta<-83^{\circ}$ where only $408 \mathrm{MHz}$ data are available. Before evaluating the distribution of the spectral in$\operatorname{dex} \beta$, we subtracted the unresolved extragalactic background and CMB absolute temperature at 408 and $1420 \mathrm{MHz}$ following Reich \& Reich (1988). The unresolved extragalactic background for the $2326 \mathrm{MHz}$ map has been estimated using the method proposed by Lawson et al. (1987). In addition a baseline offset of $-0.13 \mathrm{~K}$ for the $1420 \mathrm{MHz}$ map has been considered (see Table 2). The two maps at higher frequencies have been convolved to match the Haslam map resolution.

We carefully considered the statistical and systematic errors of each surveys in the analysis; in the definition of the overall temperature error for each pixel, we include the quoted sensitivity, $\sigma_{\text {sens }}$, the errors on the zero level, $\delta_{01}$, and calibration, $g_{\text {cal }}$, and a correction factor, $c_{\mathrm{a}}$, to account for the different area of the pixels

$c_{\mathrm{a}}=L \sqrt{\sin \theta_{i}}$,

where $L$ is the pixel side in steradian and $\theta_{i}=\pi-L i$ is the colatitude of the position of $i$ th pixel lower border. The convolution of the 1420 and $2326 \mathrm{MHz}$ maps to the resolution of the $408 \mathrm{MHz}$ map has an impact on the sensitivity; we took it into account in the error budget. The experiment that produced the $2326 \mathrm{MHz}$ map was sensitive to a linear polarization direction (Jonas et al. 1998), while the other two surveys give the total (non polarized) intensity. The available polarization surveys at low frequency do not cover large regions of those observed by the Rhodes/HartRAO $2326 \mathrm{MHz}$ telescope (for example Duncan et al. 1997, 1999) or are undersampled (Brouw \& Spoelstra 1976); thus a pixel by pixel subtraction of the linear polarized component of the $2326 \mathrm{MHz}$ map based on the polarization data is not feasible. We prefer to account for this discrepancy between the maps including the conservative term $\sigma_{\text {pol,2326 }}=20 \%$ in the error evaluation of the $2326 \mathrm{MHz}$ 

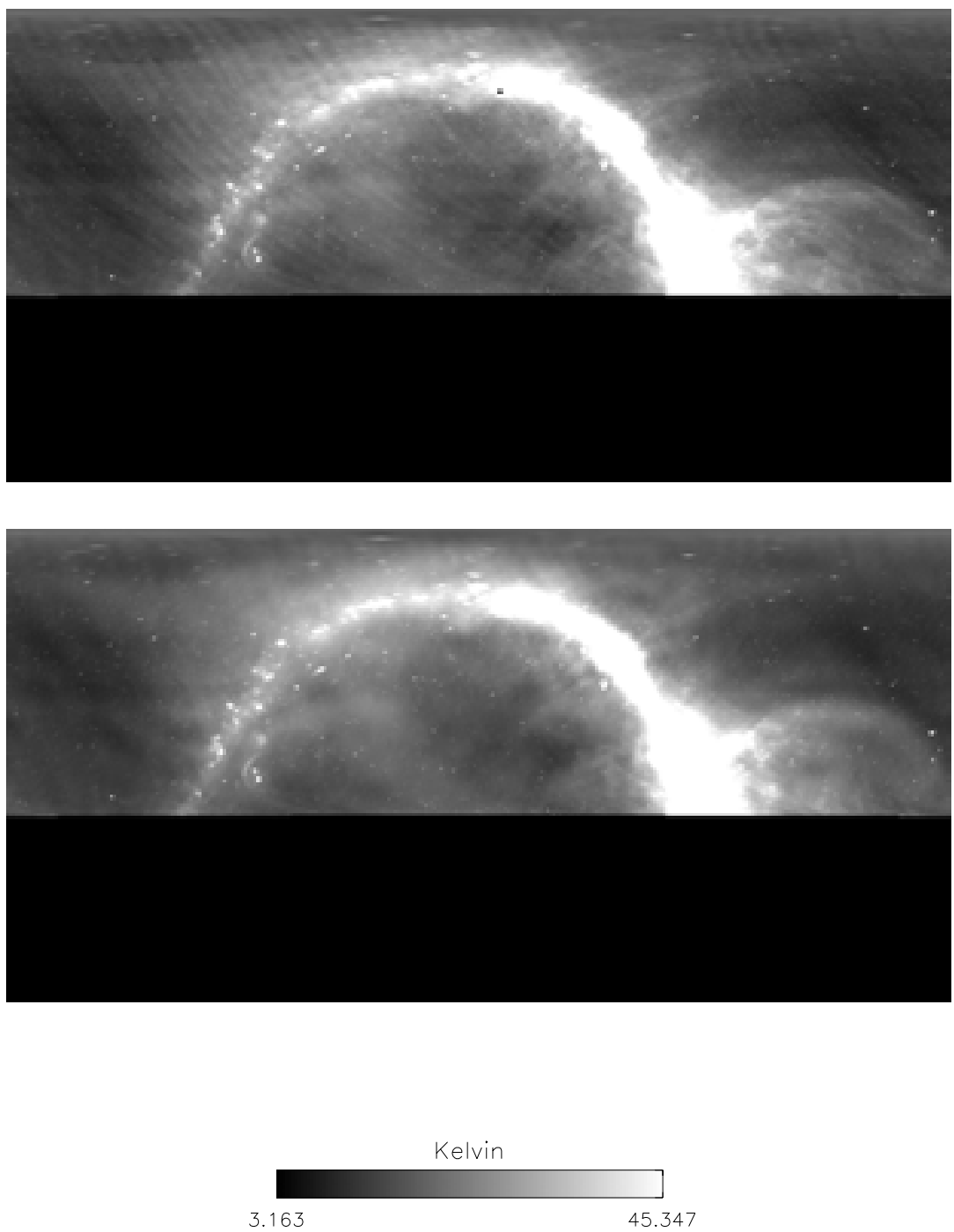

Fig. 5. Top: original 1420 MHz map (Reich 1982; Reich \& Reich 1986). Bottom: destriped 1420 MHz map (this work).

temperature (Baccigalupi et al. 2001). The final expressions for and the overall errors at the three frequencies are:

$$
\begin{aligned}
\mathrm{d} T_{408}= & {\left[\left(\sigma_{\mathrm{sens}, 408} \theta_{408}^{b} / c_{\mathrm{a}}\right)^{2}\right.} \\
& \left.+\delta_{01,408}^{2}+\left(T_{408} g_{\mathrm{cal}, 408}\right)^{2}\right]^{1 / 2} \\
\mathrm{~d} T_{1420}= & {\left[\left(\sigma_{\mathrm{sens}, 1420} \theta_{1420}^{b}\left(\theta_{1420}^{b} / \theta_{408}^{b}\right) / c_{\mathrm{a}}\right)^{2}\right.} \\
& \left.+\delta_{01,1420}^{2}+\left(T_{1420} g_{\mathrm{cal}, 1420}\right)^{2}\right]^{1 / 2} \\
\mathrm{~d} T_{2326}= & {\left[\left(\sigma_{\mathrm{sens}, 2326} \theta_{2326}^{b}\left(\theta_{2326}^{b} / \theta_{408}^{b}\right) / c_{\mathrm{a}}\right)^{2}\right.} \\
& +\delta_{01,2326}^{2}+\left(T_{2326} g_{\mathrm{cal}, 2326}\right)^{2} \\
& \left.+\left(T_{2326} \sigma_{\mathrm{pol}, 2326}\right)^{2}\right]^{1 / 2}
\end{aligned}
$$

$\log T_{v}=\alpha-\beta \log v$

where $\log a=\alpha$, the spectral index and the normalization factor and their errors are simply given by:

$\beta_{v_{1} / v_{2}}=-\frac{\log \left(T_{v_{1}} / T_{v_{2}}\right)}{\log \left(v_{1} / v_{2}\right)}$

$\alpha=\frac{\log v_{1} \log T_{v_{2}}-\log v_{2} \log T_{v_{1}}}{\log v_{1}-\log v_{2}}$,

$\sigma_{\beta}=\left|\log v_{1}-\log v_{2}\right|^{-1} \sqrt{\left(\frac{\mathrm{d} T_{1}}{T_{1} \log 10}\right)^{2}+\left(\frac{\mathrm{d} T_{2}}{T_{2} \log 10}\right)^{2}}$

where $\theta^{b}$ is the full width half maximum of the experiment at each frequency. Reminding that the relation between synchrotron radiation temperature and frequency is a power law,

$$
\begin{aligned}
\sigma_{\alpha}= & \left|\log v_{1}-\log v_{2}\right|^{-1} \\
& \times \sqrt{v_{2}^{2}\left(\frac{\mathrm{d} T_{1}}{T_{1} \log 10}\right)^{2}+v_{1}^{2}\left(\frac{\mathrm{d} T_{2}}{T_{2} \log 10}\right)^{2}} .
\end{aligned}
$$

$$
T_{v}=a v^{-\beta},
$$

(3) where 1 and 2 are two generic frequencies. 

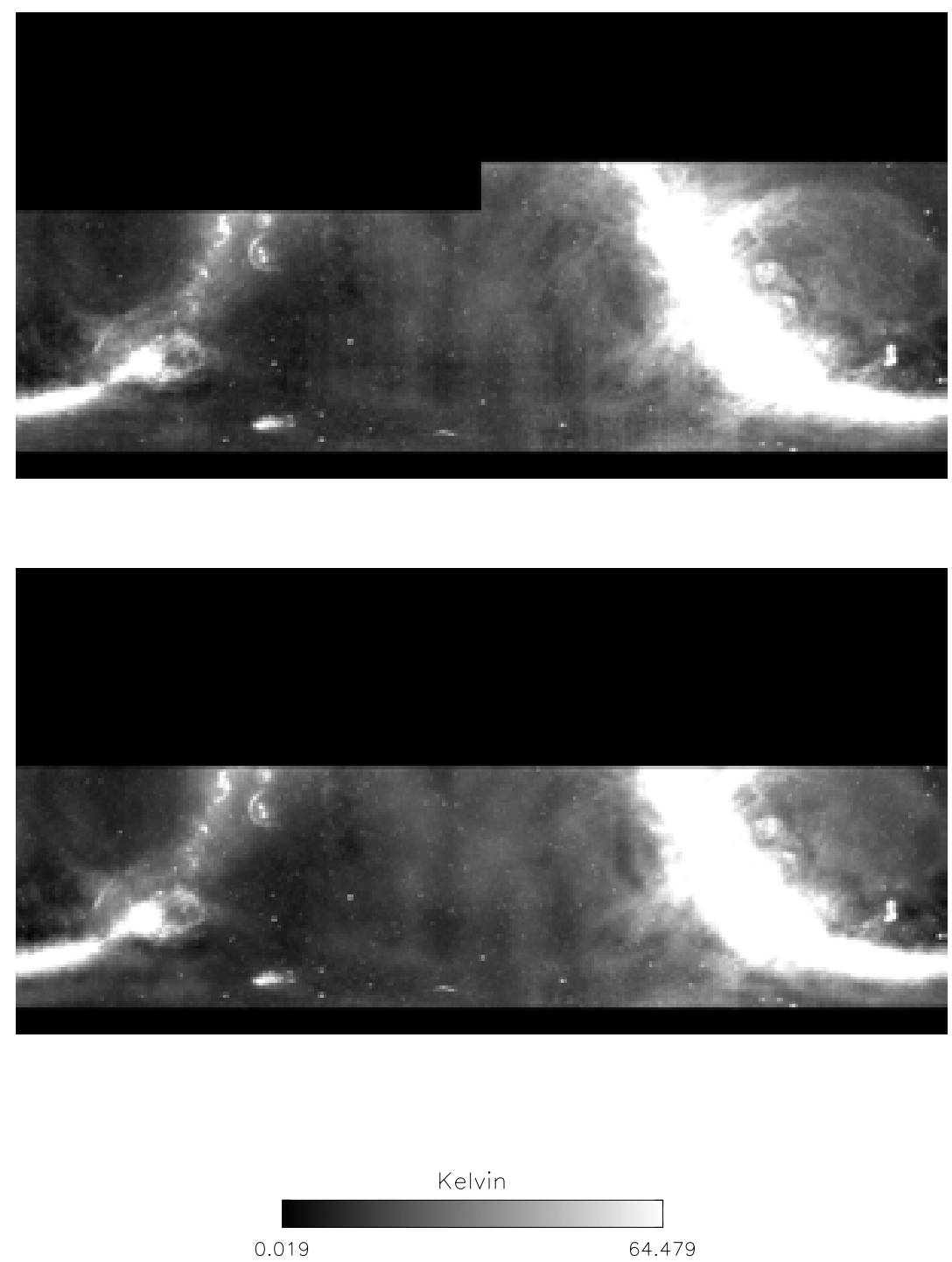

Fig. 6. Top: original $2326 \mathrm{MHz}$ map (Jonas et al. 1998). Bottom: destriped $2326 \mathrm{MHz}$ map (this work). For an easier division in relatively large squared patches, the region $13^{\circ}<\delta<32^{\circ}$ of the $2326 \mathrm{MHz}$ map has not been included in the data analysis steps described in Sects. 3.1 and 3.2.

In the central region where the three maps overlap, we perform a least square fit to evaluate the $\beta, \alpha$ and their errors (Press et al. 1992) assuming a constant $\beta$ between 408 and $2326 \mathrm{MHz}$. We repeated the same analysis including only the statistical errors in the error budget. We estimated the influence of the stripes threshold choice on the spectral index distribution: the mean error is $\sim 1 \%$, thus negligible in the error budget.

As we expect, the average spectral index and dispersion are not significantly affected by destriping, while substantial changes are produced on a pixel by pixel basis (up to $\Delta \beta \sim 0.4$ ). Figure 7 shows the difference $\Delta \beta$ between the spectral indices evaluated with destriped and original maps.

\subsection{Frequency variation analysis}

The steepening of the synchrotron spectrum with increasing frequency has to be taken into account in any estimate of the Galactic radio emission. We tested the assumption that the spectral index between 408 and $2326 \mathrm{MHz}$ does not change significantly when evaluated with different combinations of the three surveys. In the overlapping region, where data at the three frequencies are available, we compare $\beta_{408,1420}$ with $\beta_{1420,2326}$. Figure 8 shows the distribution of the difference between the spectral index evaluated with 408 and $1420 \mathrm{MHz}$ data, $\beta_{408,1420}$, and the spectral index evaluated with the 1420 and $2326 \mathrm{MHz}$ data, $\beta_{1420,2326}$. The shape of the distribution indicates that there is not a clear steepening of $\beta$ when using these surveys. Moreover, we compare pixel by pixel the distribution of the difference with the standard deviation $\sigma_{\beta}$, where, for each pixel $\sigma_{\beta}^{2}=\sigma_{\beta(408,1420)}^{2}+\sigma_{\beta(1420,2326)}^{2}$ : only for the $5 \%$ of the pixels the difference between the spectral indices is larger than the estimated error. The same analysis performed with the statistical errors only shows that for the $25 \%$ of the pixels the difference is larger than $\sigma_{\beta, \text { stat }}$. We conclude that, generally, the estimated error is larger than the frequency variation of the spectral index $\beta$ between 1420 and $2326 \mathrm{MHz}$. This motivates the idea to jointly use, where possible, all the three surveys to derive a single map of spectral indices and normalization factors in the frequency range $0.408-2326 \mathrm{MHz}$. As discussed in Sect. 2, these surveys are affected by zero level and calibration uncertainties; 
Table 2. Calibration errors $\sigma_{\text {cal }}$, zero level errors $\sigma_{01}$ and baseline corrections from the original experiments at 408,142 and $2326 \mathrm{MHz}$; extragalactic background for the 408 and $1420 \mathrm{MHz}$ maps have been estimated by Lawson et al. (1987), for the $2326 \mathrm{MHz}$ map in this work following the same procedure.

\begin{tabular}{|c|cccc|}
\hline \hline $\begin{array}{c}\text { Survey } \\
\text { Frequency (MHz) }\end{array}$ & $\begin{array}{c}\text { calibration } \\
\text { error (per cent) }\end{array}$ & $\begin{array}{c}\text { Zero level } \\
\text { error (K) }\end{array}$ & $\begin{array}{c}\text { Baseline } \\
\text { correction }\end{array}$ & $\begin{array}{c}\text { Extragalactic } \\
\text { background (K) }\end{array}$ \\
\hline 408 & 10 & 3 & - & 5.92 \\
1420 & 5 & 0.5 & -0.13 & 2.83 \\
2326 & 5 & 0.080 & - & 2.75 \\
\hline
\end{tabular}
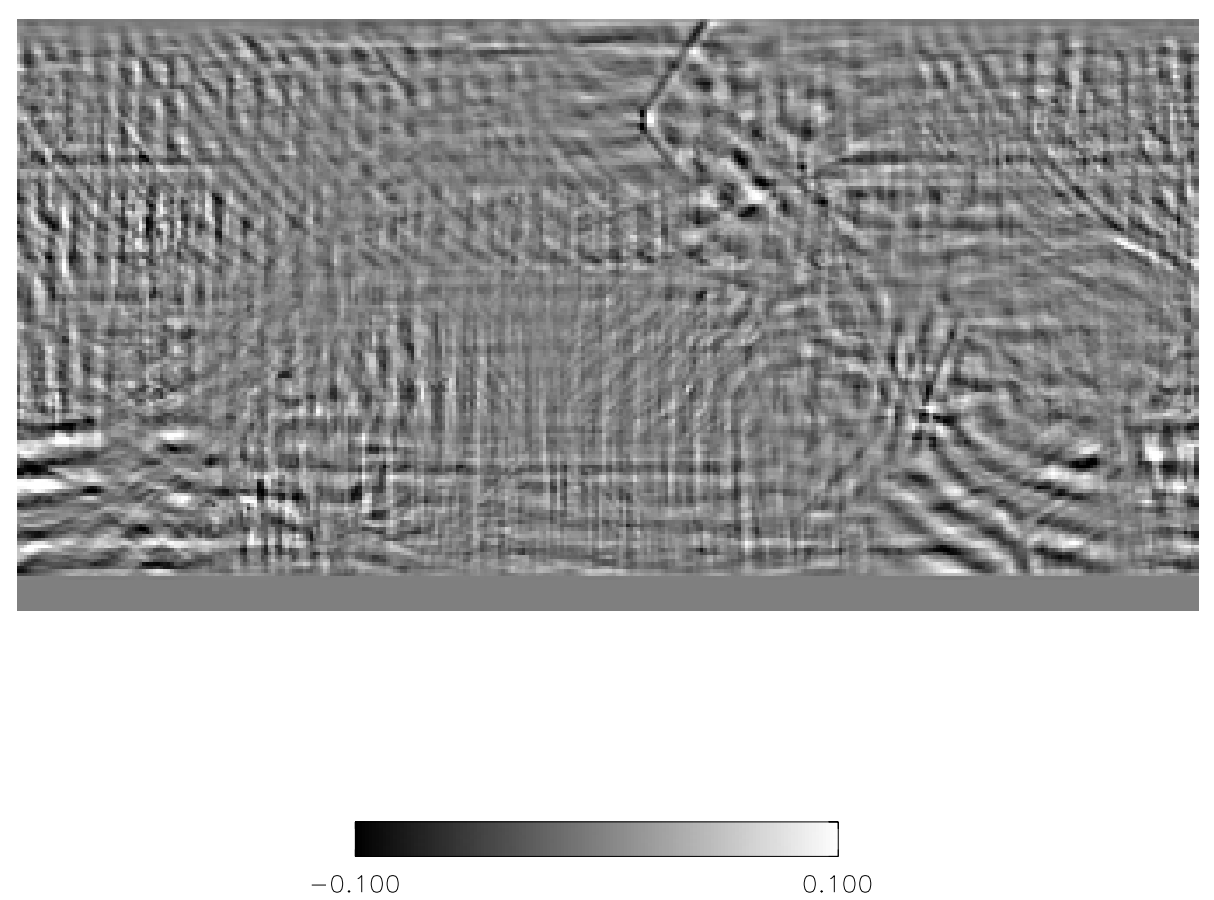

Fig. 7. Map of the differences $D \beta$ between the spectral index evaluated with the destriped and the original maps. Features corresponding to stripes are evident.

the latter are particularly relevant at $2326 \mathrm{MHz}$. In the next subsection we discuss an attempt to reduce, at least in part, the impact of these uncertainties in the joint analysis of the three surveys.

\subsection{Discontinuities}

As one might expect, the $\beta$ and $\alpha$ maps show two horizontal lines of discontinuity corresponding to the borders of the maps at 1420 and $2326 \mathrm{MHz}$. Of course these discontinuities arise from measurements and inter-calibration errors and do not have any astrophysical meaning.

A smoothing of the data to a lower resolution can be easily applied to eliminate the appearence of this effect but would not eliminate its causes. The same effect has been observed by Giardino et al. (2002) who combined the 408, 1420 and $2326 \mathrm{MHz}$ maps to obtain a large angular resolution $\left(10^{\circ}\right)$ map of $\beta$. To eliminate the discontinuities, they tried different

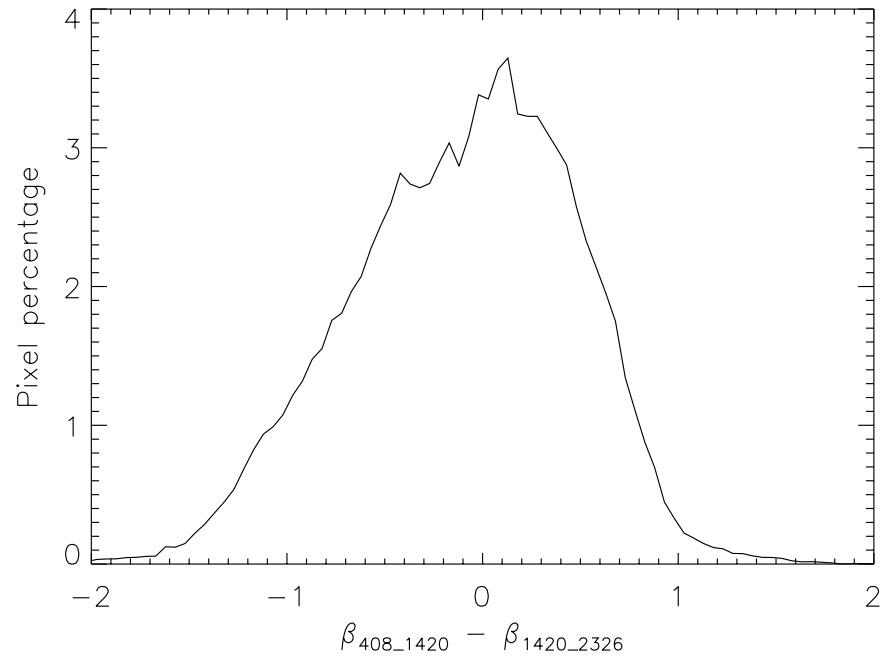

Fig. 8. Distribution of the differences $\beta_{408,1420}-\beta_{1420,2326}$. The binsize is 0.01 . 

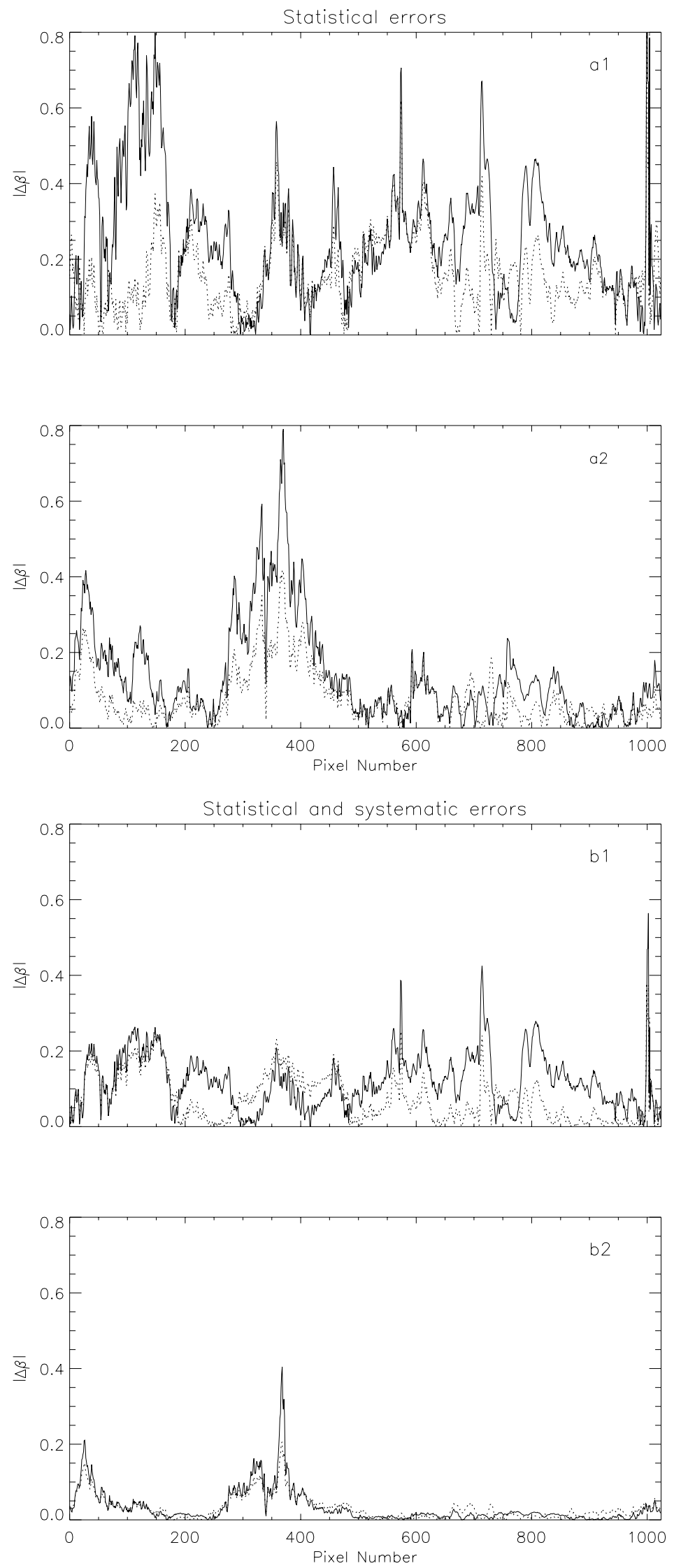

Fig. 9. Differences in $\beta$ in the case of statistical error only a) and systematics included b); pixel number [0-1024] corresponds to right ascension $\left[-180^{\circ}, 180^{\circ}\right]$. The curves show the up (a1) and b1)) and down (a2) and b2)) discontinuities before (solid) and after (dotted) minimization. It is worth to remind that the values $\Delta \beta$ and $\Delta \alpha$ before minimization depend on the kind of errors included in the analysis, via their influence on the fit in the case we use data at all the three frequencies. 

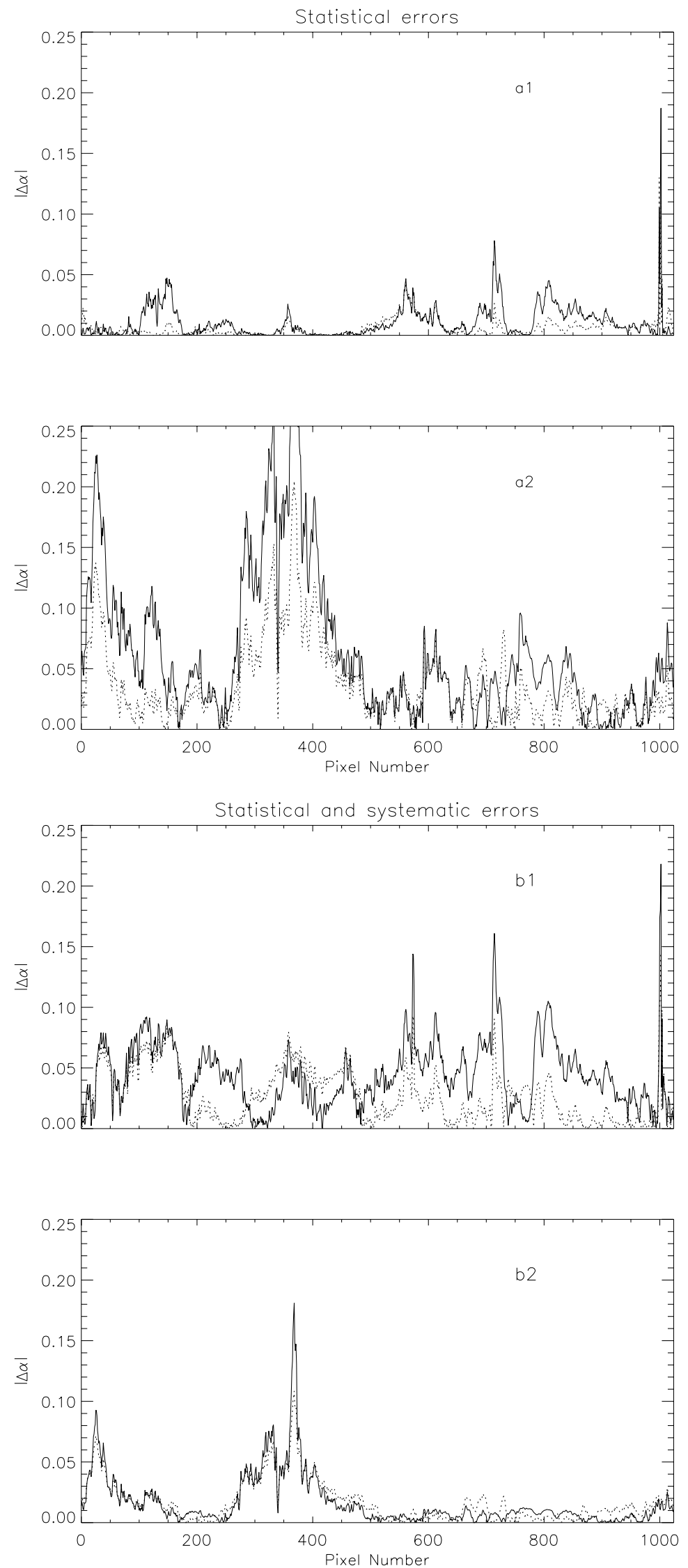

Fig. 10. Differences in $\alpha$ in the case of statistical error only a) and systematics included b). The curves show the up (a1) and b1)) and down (a2) and b2)) discontinuities before (solid) and after (dotted) minimization. See comments for Fig. 9. 

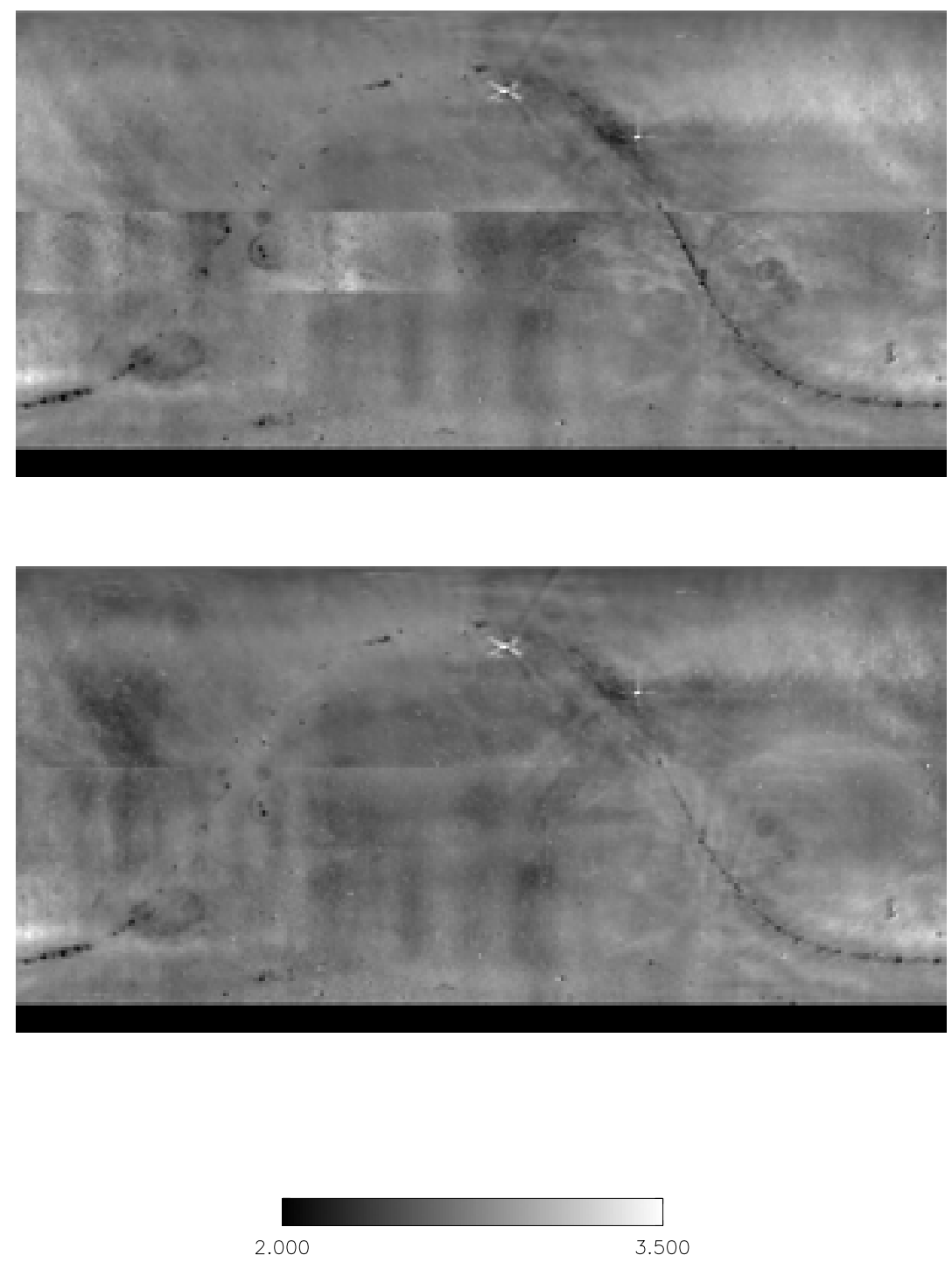

Fig. 11. Maps of the synchrotron spectral index $\beta$ after minimization. Top: the analysis has been carried out with temperature statistical errors only. Bottom: systematic errors have been included.

techniques looking for a baseline offset for one data set, with no improvement. In the overlapping region, they set the spectral index to a weighted average of $\beta_{408,1420}$ and $\beta_{408,2326}$; the choice of the weights ensures that the three different sky regions smoothly merge into each other. By convolving the data sets to $10^{\circ}$ resolution, the stripes and discontinuities effects were averaged out. In this work we are interested in the high resolution information of the spectral index; after the destriping procedure discussed in Sect. 3, we investigate to what extent the discontinuities in the $\alpha$ and $\beta$ maps could be accounted for within the quoted measurements systematic errors of the three surveys (see Table 2), i.e., gain and zero-level errors. One should be aware that these uncertainties include a number of effects related to the details of the scanning strategy and calibration procedure adopted in the observations. All three surveys are obtained by merging together different sky patches with zero levels somewhat different from one another. In the merging phase of the map production and as a-posteriori analysis, attempts were made to minimise discontinuities between different patches (e.g., Reich \& Reich 1988). In addition, these surveys have been calibrated by convolution to sky horn absolute measurements, typically obtained at fixed declinations. With increasing distance from the absolute calibration data, the uncertainty in the zero level could increase due to residual baseline gradients. Therefore using zero-level errors as "baseline errors" is equivalent to the assumption that the underlying constant component is of the same order as the total uncertainty. Although not ideal, we follow this assumption since it is the only practical possibility at hand. A deeper analysis would require the availability of the original raw data of each survey to perform a complete reanalysis, a task which is outside the scope of this work.

We searched for the combination of six parameters (three pairs of parameters, calibration factor $g_{\text {cal }}$ and zero level correction $\delta_{01}$, each for each frequency map) to rescale the maps to account for their zero level and calibration errors with the aim to minimize the difference across the discontinuities. Of course, the assumption that these three couples of parameters 

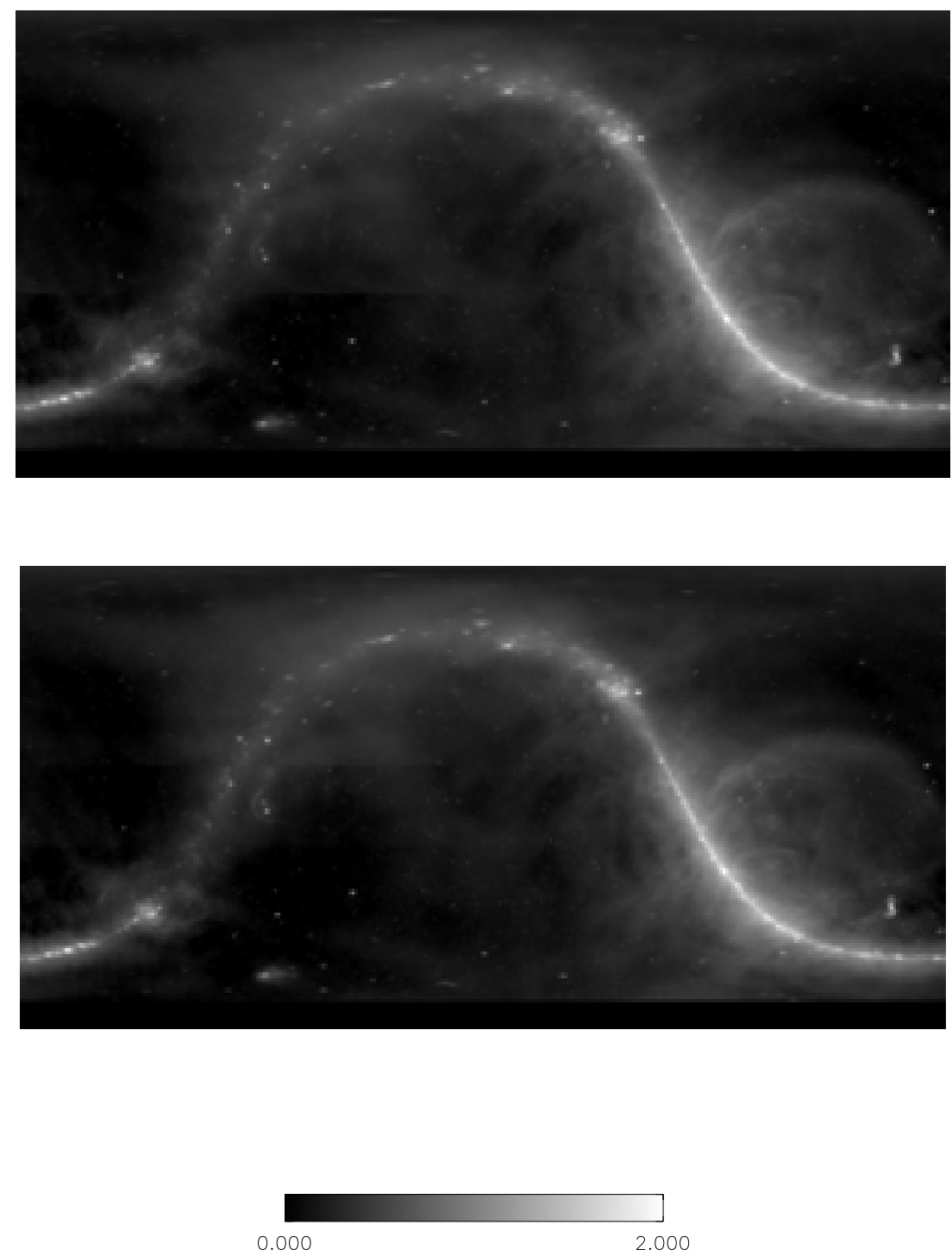

Fig. 12. Maps of the normalization factor $\alpha$ after minimization. Top: the analysis has been carried out with temperature statistical errors only. Bottom: systematic errors have been included.

are constant over the entire survey is an over-simplification; in fact it is very hard, if not impossible, to recover the real pattern of the variations of these parameters from the considered data products (see below for an attempt in this direction). The six parameters influence the $\beta$ and $\alpha$ values through the optimized temperatures of the maps:

$T_{v \text { opt }}=T_{v} g_{\mathrm{cal}}+\delta_{01}$

We adopted the following $\chi^{2}$-like form of the functional to be minimized:

$$
\begin{aligned}
\chi^{2}= & \sum_{i=1}^{N} \frac{\left(\beta_{2 \mathrm{~d}}(i)-\beta_{3 \mathrm{~d}}(i)\right)^{2}}{\sigma_{\beta_{2 \mathrm{~d}}}^{2}(i)+\sigma_{\beta_{3 \mathrm{~d}}}^{2}(i)}+\frac{\left(\beta_{2 \mathrm{u}}(i)-\beta_{3 \mathrm{u}}(i)\right)^{2}}{\sigma_{\beta_{2 \mathrm{u}}}^{2}(i)+\sigma_{\beta_{3 \mathrm{u}}}^{2}(i)} \\
& +\frac{\left(\alpha_{2 \mathrm{~d}}(i)-\alpha_{3 \mathrm{~d}}(i)\right)^{2}}{\sigma_{\alpha_{2 \mathrm{~d}}}^{2}(i)+\sigma_{\alpha_{3 \mathrm{~d}}}^{2}(i)}+\frac{\left(\alpha_{2 \mathrm{u}}(\mathrm{i})-\alpha_{3 \mathrm{u}}(\mathrm{i})\right)^{2}}{\sigma_{\alpha_{2 \mathrm{u}}}^{2}(i)+\sigma_{\alpha_{3 \mathrm{u}}}^{2}(i)}
\end{aligned}
$$

where "u" and " $\mathrm{d}$ " refers to the up and down discontinuities respectively, the low indices 2 and 3 refers to $\beta$ and $\alpha$ evaluated by exploiting two or three frequency maps, and the sum is carried out over $i$ th azimuthal position of all the $N$ pixels of the top and bottom border lines of the overlapping region. The minimization has been carried out by using the MINUIT package of the CERN library ${ }^{6}$. Different approaches have been tested in this minimization: we left all the parameters free, we kept fixed each of them (e.g. $\delta_{01}=0$ and $g_{\text {cal }}=1$ or viceversa for a given frequency map) while leaving the others free; we varied each of them at one time keeping fixed the others, and finally, we varied the parameters $\delta_{01}$ and $g_{\text {cal }}$ of only a given frequency map by keeping the "original" parameters at the other frequencies ${ }^{7}$. By observing that two frequency maps (at 408 and $2326 \mathrm{MHz}$ ) show, before destriping, almost vertical striations due to the scanning direction, we implemented also a modified version of this minimization technique: instead of searching for six overall parameters over the whole maps, we searched for six azimuthal (e.g. as function of the right ascension) profiles of

\footnotetext{
${ }^{6} \mathrm{http}: / /$ cern.web.cern.ch/CERN/

7 Of course the choice $g_{\text {cal }}=1$ and $\delta_{01}=0$ corresponds to the "original" map.
} 

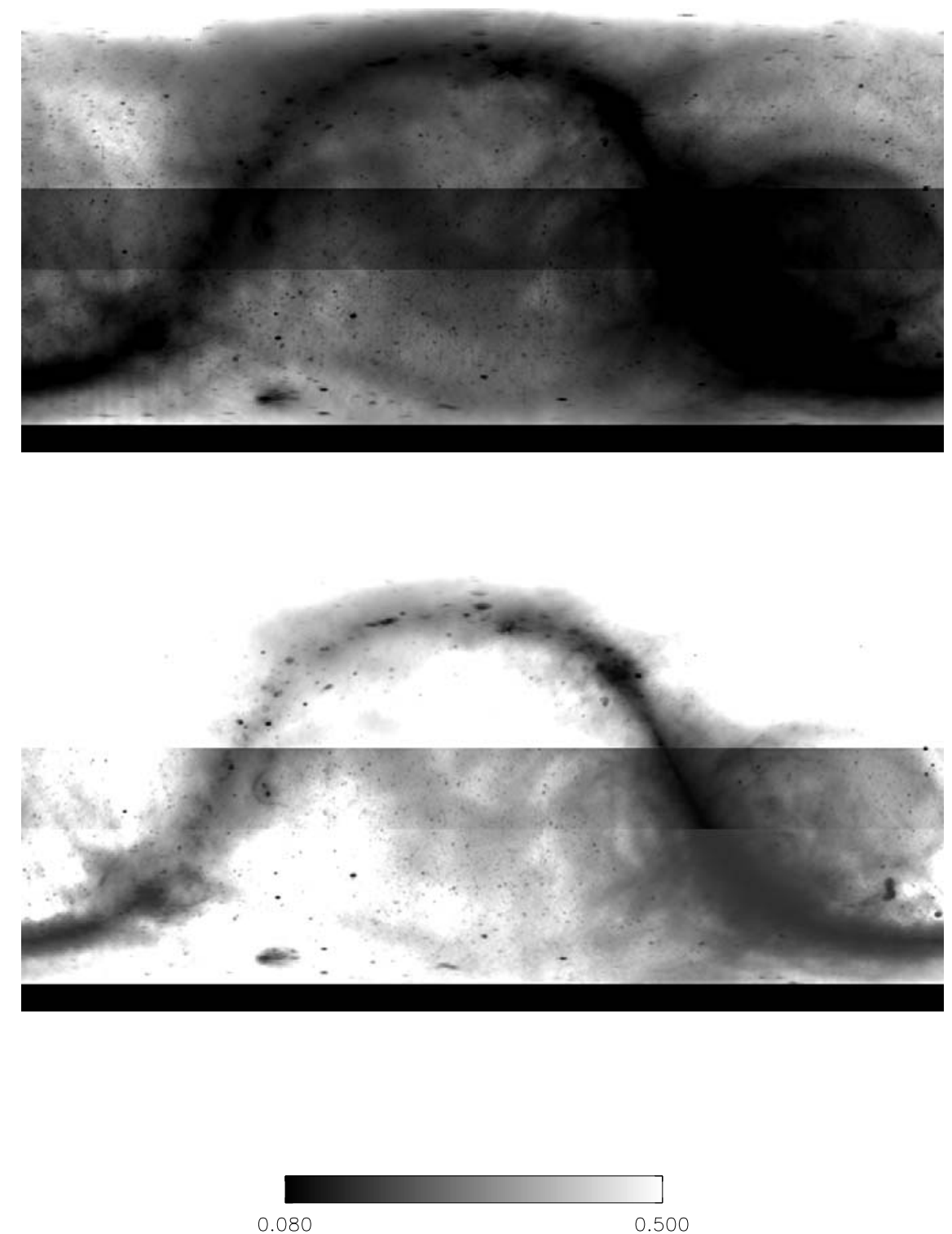

Fig. 13. Maps of the synchrotron spectral index errors $\sigma_{\beta}$ after minimization. Top: the analysis has been carried out with temperature statistical errors only. Bottom: systematic errors have been included.

the three pairs of $\delta_{01}$ and $g_{\mathrm{cal}}$ by implementing, at each $i$ th azimuthal position, an overall minimization of the discrepances between the values obtained for $\beta$ and $\alpha$ by using three frequency maps or two combinations of only two frequency maps for all the pixels at the same $i$ th azimuthal position but at the different declinations where three frequency maps are available ( $\simeq 90$ pixels at each $i$ th azimuthal position). This approach should in principle better account for possible azimuthal variations $\delta_{01}$ and $g_{\text {cal }}$ over the maps in the case in which they do not show a significant declination dependence. Unfortunately, this method, although quite effective in reducing horizontal discontinuities, introduces undesired vertical discontinuities, suggesting for complex patterns of $\delta_{01}$ and $g_{\text {cal }}$ whose recovery cannot clearly be derived by using such kinds of minimization approaches.
We then adopted the overall minimization approach described by the Eq. (10) searching for the optimized set of the above six parameters.

Of course, the best result from the point of view of the reduced $\chi^{2}$ and of the minimization of discontinuities, is obtained leaving the all six parameters free to vary. On the other hand, it is clear that we need to keep them inside their corresponding experimental quoted errors. This requirement can be relaxed for the parameter $g_{\text {cal }}$ referring to the map at $2326 \mathrm{MHz}$ because of the uncertainty related to the polarization, as discussed in the previous subsection, which could account for variations of $\approx 10 \%$. Moreover, the $2326 \mathrm{MHz}$ data have been calibrated using $2 \mathrm{GHz}$ data (Bersanelli et al. 1994) scaled with a spectral index of 2.75; the relaxed requirement can take into account also the bias introduced in the determination of the spectral index distribution by the calibration procedure. 

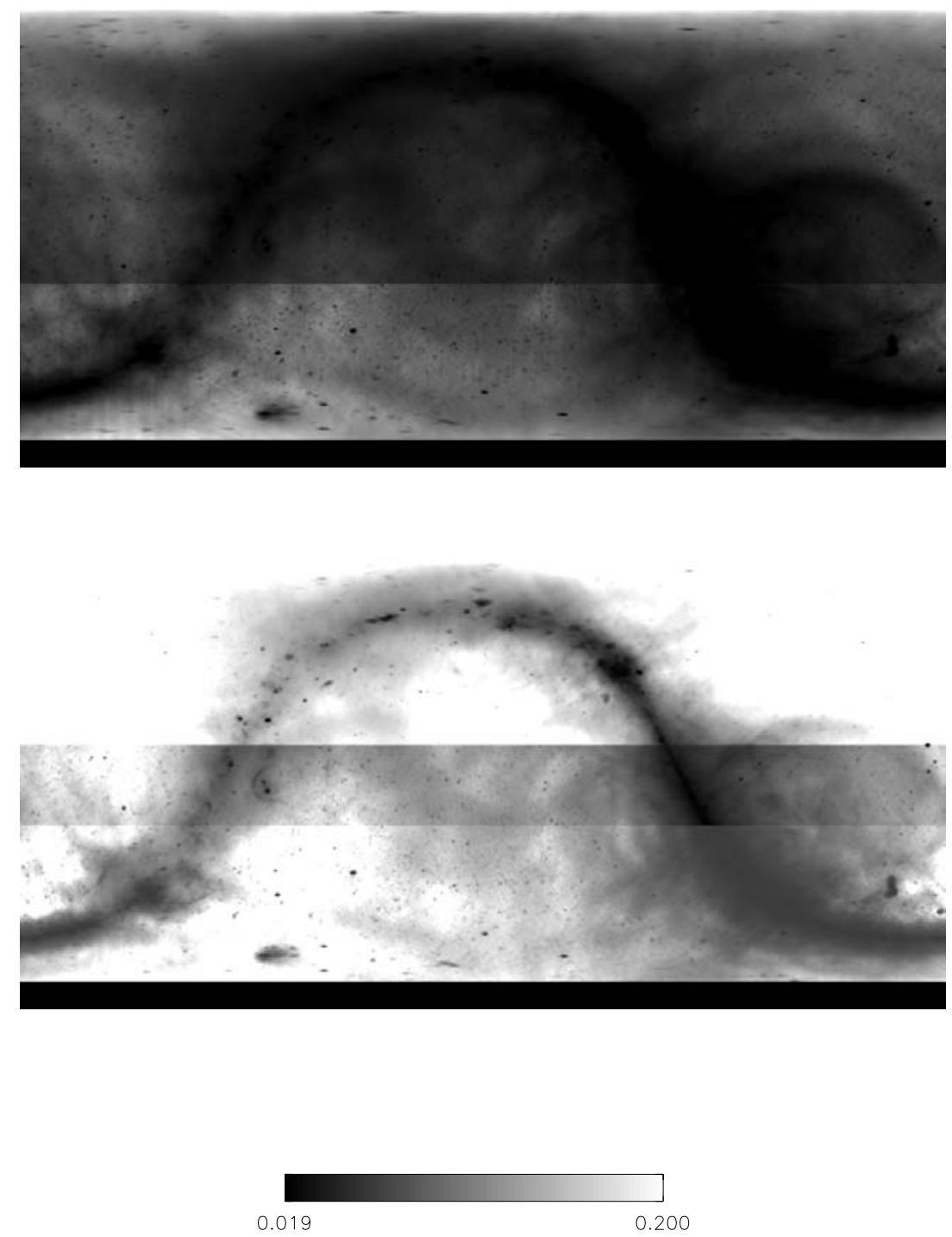

Fig. 14. Maps of the normalization factor errors $\sigma_{\alpha}$ after minimization. Top: the analysis has been carried out with temperature statistical errors only. Bottom: systematic errors have been included.

Table 3. Calibration factors and zero level corrections for the three maps as recovered after the minimization for statistical errors only and with systematic errors includes. The $\chi^{2} /$ d.o.f. is also shown.

\begin{tabular}{|l|cc|}
\hline \hline & $\begin{array}{c}\text { statistical } \\
\text { errors only }\end{array}$ & $\begin{array}{c}\text { statistical + systematic } \\
\text { errors }\end{array}$ \\
\hline$g_{\mathrm{cal}, 408}$ & 0.877 & 0.973 \\
$g_{\mathrm{cal}, 1420}$ & 1.100 & 1.066 \\
$g_{\mathrm{cal}, 2326}$ & 0.827 & 0.793 \\
$\delta_{01,408}$ & $3.432 \mathrm{~K}$ & $0.676 \mathrm{~K}$ \\
$\delta_{01,1420}$ & $-0.0410 \mathrm{~K}$ & $-0.0267 \mathrm{~K}$ \\
$\delta_{01,2326}$ & $0.0366 \mathrm{~K}$ & $0.0357 \mathrm{~K}$ \\
$\chi^{2} /$ d.o.f. & 14.381 & 0.204 \\
\hline
\end{tabular}

We carried out the above minimization with $\delta_{01}$ and $g_{\text {cal }}$ inside their quoted $2 \sigma$ errors for the frequency maps at 408 and $1420 \mathrm{MHz}$, and with $\delta_{01}\left(g_{\mathrm{cal}}\right)$ inside its quoted $2 \sigma$ error $(5 \sigma$ error) for the frequency map at $2326 \mathrm{MHz}$.
We have repeated this analysis by including or not the contribution of systematics in the error budget, as discussed in the previous subsection.

In addition, we have tested that the results do not depend on the adopted range of allowed parameter values when the systematic errors are included. On the other hand, neglecting the systematic errors, the minimization tends to give a best fit value of $g_{\text {cal }}$ at $1420 \mathrm{MHz}$ out of its quoted $2 \sigma$ error. In this case, we have then repeated the minimization by constraining $g_{\mathrm{cal}}$ at $1420 \mathrm{MHz}$ within different ranges (within 1,2 and $5 \sigma$ ) and keeping the allowed ranges for the other variables as before. We find an impact of a few percent on average and always less than $10 \%$ (with a change less than $1 \%$ for half of the pixels) on the maps of $\beta$ and $\alpha$. In Table 3 we show the six parameters after minimization. The results obtained by including or not systematic errors are in agreement within $1 \sigma$ error in each variable of the fit. The parabolic errors quoted for each recovered parameter from the minimization code are much smaller than the corresponding experimental uncertainties and discrepancies found 

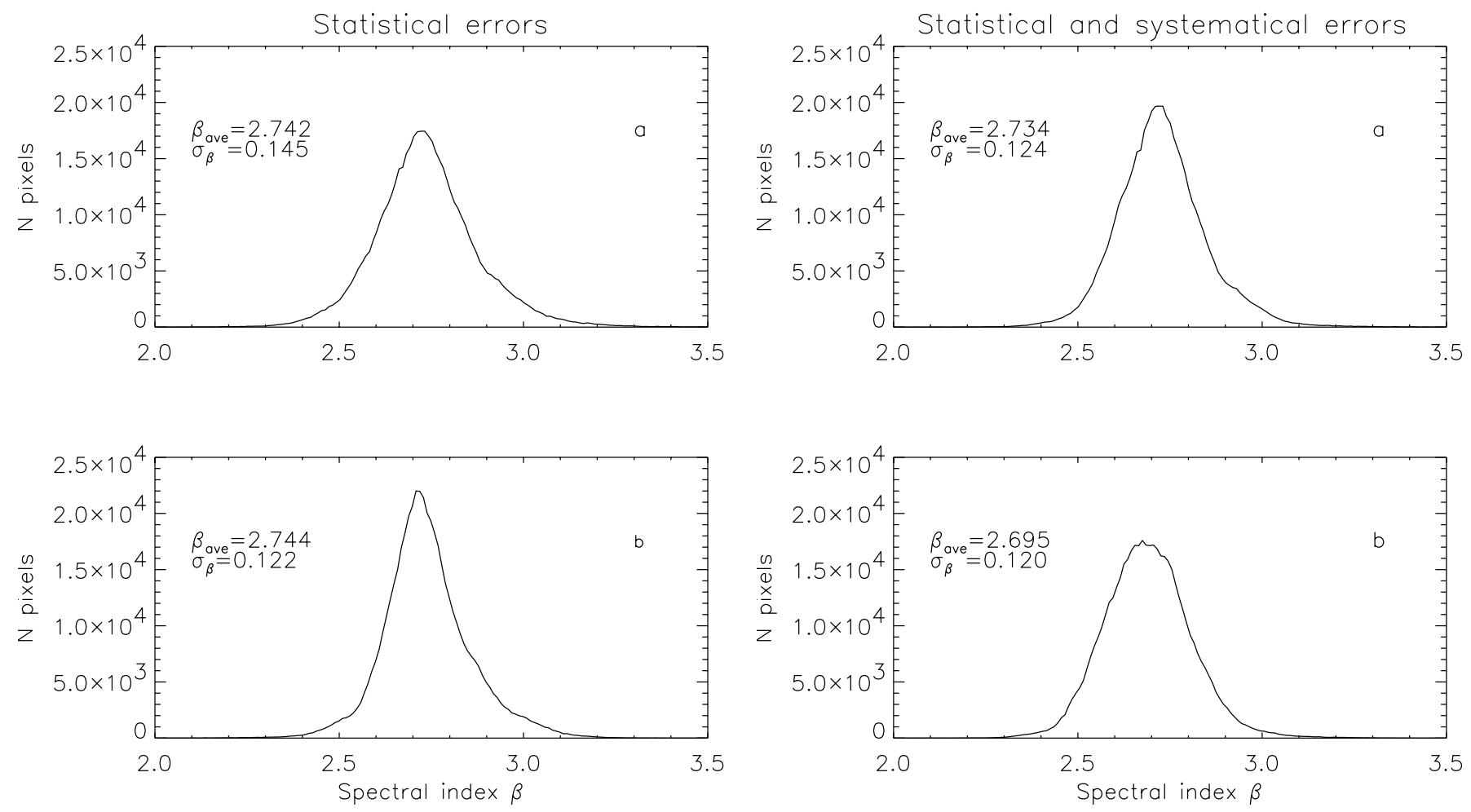

Fig. 15. Analysis with statistical errors only. The histogram of the spectral indices before a) and after b) minimization are shown together with their average and dispersion.

including or not systematics, and we avoid to report them, being clearly "unphysical" ${ }^{8}$. As expected, the $\chi^{2} /$ d.o.f. is larger (smaller) than unity when systematic errors are neglected (included) in the analysis. It is worth to point out that the parameters in Table 3 have to be considered not as the "real" correction to the experimental values of baseline and calibration of each survey, individually considered, but as the result of a procedure devoted to obtain more physically meaningful distributions of spectral index and normalization factor from the joint exploitation of the three surveys. This is particulary clear in the case of the $2326 \mathrm{MHz}$ map where, to allow the comparison of a linearly polarized survey with the intensity data, the recovered calibration parameter has been found well out the experimental values.

The differences, $\Delta \beta$ and $\Delta \alpha$, in correspondence to the discontinuities, before and after having applied the described minimization neglecting or including the systematics, are shown in Figs. 9 and 10. Note that the differences are evaluated between the two adjacent lines across each discontinuities, while the minimization is carried out with the values of $\beta$ evaluated with two or three frequencies in each border line of the overlapping region. The algorithm has the same qualitative effect if we consider only statistical or also systematic errors.

\footnotetext{
${ }^{8}$ Clearly, in the maps of the final error on $\beta$ and $\alpha$ the contribution of these parabolic errors is also negligible.
}

Fig. 16. Analysis with statistical and systematic errors. The histogram of the spectral indices before a) and after b) minimization are shown together with their average and dispersion.

\subsection{Results}

The resulting maps of $\beta, \alpha$ and their errors are shown in Figs. 11-14.

From Figs. 9 and 10 it is clear that discontinuities do not completely disappear; they are reduced, but the minimization procedure is not able to account for the full complexity of the real experimental drifts of the six parameters. The increasing of $\sigma_{\beta}$ and $\sigma_{\alpha}$ with declination are explained considering the correction factor $c_{\mathrm{a}}$ appearing in Eq. (2) to account for nonequal area pixels.

The distributions of the spectral index $\beta$ are shown in Figs. 15 and 16; we evaluated the average spectral index and the dispersion before and after minimization, as summarized in Table 4; the effect of minimization is to slightly change both parameters. The values well agree with those found by Giardino et al. (2002) of $\bar{\beta}_{480,1420}=2.78 \pm 0.17$ and $\bar{\beta}_{480,2326}=$ $2.75 \pm 0.12$. The minimization procedure does not alter significantly the pixel by pixel values of $\beta$ : typically, a variation greater than 0.1 occurs for a few $\%$ of the pixels.

We have estimated the contribution of point sources on the $\beta$ distribution as follows: we subtracted the point sources from the destriped maps (with the same technique explained in Sect. 3) and performed all the same steps on the analysis. The main results is a sensible reduction of the low spectral index tail in the distribution ${ }^{9}$; on the other hand, when extended

\footnotetext{
${ }^{9}$ The high $\beta$ tail comes from extended sources not removed by the source selection method.
} 
Table 4. Mean values and dispersion of the spectral index distributions. The percentage of pixels with $\beta<2$ and $>3.5$ is shown.

\begin{tabular}{|l|c|cc|}
\hline \hline & & $\begin{array}{c}\text { statistical } \\
\text { errors only }\end{array}$ & $\begin{array}{c}\text { statistical + systematic } \\
\text { errors }\end{array}$ \\
\hline before minimization & $\bar{\beta} \pm \sigma_{\beta}$ & $2.742 \pm 0.145$ & $2.734 \pm 0.124$ \\
& \%pixels with $\beta<2$ & 0.031 & 0.015 \\
& \%pixels with $\beta>3.5$ & 0.066 & 0.05 \\
\hline \multirow{2}{*}{ after minimization } & $\bar{\beta} \pm \sigma_{\beta}$ & $2.744 \pm 0.122$ & $2.695 \pm 0.120$ \\
& \%pixels with $\beta<2$ & 0.022 & 0.009 \\
& \%pixels with $\beta>3.5$ & 0.012 & 0.012 \\
\hline
\end{tabular}

regions are considered, this procedure can sensibly smoothing the outer bordes of the structure.

The incidence of free-free diffuse contribution has been estimated as follows. We scaled the WMAP free-free template (Bennett et al. 2003) at 408, 1420 and $2326 \mathrm{MHz}$ and subtracted from the destriped maps. We obtained a variation of $\beta$ larger than 0.1 for less than $8 \%$ of the pixels; this difference is inside the deviations illustrated in Table 4 except for a few percentage of the pixels. Thus, we can conclude that the diffuse free-free emission does not sensibly affect the distributions obtained in this work.

We can say that the diffuse Galactic maps resulting from this work are representative for synchrotron spectral index distribution and synchrotron normalization factor. Point sources contribution is limited to a small number of pixels that could be isolated and removed to extrapolate the maps at high frequency and obtain synchrotron emission templates.

\section{Discussion and conclusions}

We analysed the contribution of systematic uncertainties in the diffuse Galactic emission surveys at 408, 1420 and $2326 \mathrm{MHz}$, cleaning the striping effect arising in combination with the scanning strategy.

We used the low frequency destriped maps to obtain the spectral index and normalization factor distributions over the whole observed sky.

We performed a minimization technique over the calibration and zero level errors of the surveys to reduce the discontinuities in the $\alpha$ and $\beta$ maps in correspondence to the borders of the 1420 and $2326 \mathrm{MHz}$ maps. The artificial lines of discontinuities are reduced but not eliminated, suggesting for a complex pattern of $\delta_{01}$ and $g_{\text {cal }}$.

We discussed the spectral index distribution recovered; by estimating the contribution of point sources and diffuse freefree emission, we concluded that the spectral index distribution is representative for the diffuse Galactic synchrotron emission between 408 and $2326 \mathrm{MHz}$.

The new results of the WMAP experiment give a quite precise picture of the Galactic emissions between $\simeq 23$ and $94 \mathrm{GHz}$ (Bennett et al. 2003). The theory predicts a break in the synchrotron spectrum of the Milky Way at $22 \mathrm{GHz}$ (Voelk 1989), observed in the WMAP data. In the synchrotron spectral index map between 0.408 and $23 \mathrm{GHz}$ produced by the WMAP team, emission from the Galactic plane is dominant (the North Galactic Spur is much less evident than at radio frequencies), since, with increasing frequency, the flat components of the spectrum become more important than the steep components. For example, the fact that in the WMAP data the North Galactic Spur is much less evident than at radio frequencies shows clearly that any template of the Galactic emission is frequency dependent. This puts more interest in the comparison between the new data and those derived from radio surveys.

Acknowledgements. We warmly thank D. P. Finkbeiner for deep explanation of the destriping concept and Roger Hoyland for useful discussions. Some of the results in this paper have been derived using the HEALPix (Gòrski et al. 1999). We wish to warmly thank the referee for useful and constructive comments.

\section{References}

Baccigalupi, C., Burigana, C., Perrotta, F., et al. 2001, A\&A, 372, 8

Banday, A. J., Dickinson, C., Davies, R. D., Davis, R. J., \& Gòrski, K. M. 2003, MNRAS, accepted [astro-ph/0302181]

Bennett, C. L., Hill, R. S., Hinshaw, G., et al. 2003, ApJS, 148, 97

Bersanelli, M., Bensadoun, M., de Amici, G., et al. 1994, ApJ, 424, 517

Brouw, W. N., \& Spoelstra, T. A. Th. 1976, A\&AS, 26, 129

Cappellini, B., Burigana, C., \& Platania, P. 2003, Internal Report IASF-BO/CNR 365/2003, PL-LFI-IASFbo-MA-003, February

Cappellini, B., Burigana, C., \& Platania, P. 2003, Internal Report IASF-BO/CNR 372/2003, PL-LFI-IASFbo-MA-005 July

Davies, R. D., Watson, R. A., \& Gutierrez, C. M. 1996, MNRAS, 278, 925

Duncan, A. R., Haynes, R. F., Jones, K. L., \& Stewart, R. T. 1997, MNRAS, 291, 279

Duncan, A. R., Reich, W., Reich, P., \& Fürst, E. 1999, A\&A, 350, 447

Giardino, G., Banday, A. J., Górski, K. M., et al. 2002, A\&A, 387, 82

Górski, K. M., Hivon, E., \& Wandelt, B. D. 1999, Proceedings of the MPA/ESO Conference on Evolution of Large-Scale Structure: from Recombination to Garching, ed. A. J. Banday, R. K. Sheth, \& L. Da Costa, 37 
Haslam, C. G. T., Stoffel, H., Salter, C. J., \& Wilson, W. E. 1982, A\&AS, 47, 1

Jonas, J., Baart, E. E., \& Nicolson, G. D. 1998, MNRAS, 297, 997

Keihänen, E., Kurki-Suonio, H., Poutanen, T., Maino, D., \& Burigana, C. 2003, A\&A, submitted [astro-ph/0304411]

Lawson, K. D., Mayer, C. J., Osborne, J. L., \& Parkinson, M. L. 1987, MNRAS, 225, 307

Maino, D., Banday, A. J., Baccigalupi, C., Perrotta, F., \& Górski, K. M. 2003, MNRAS, 344, 544
Press, W. H., Teukolsky, S. A., Vetterling, W. T., \& Flannery, B. P. 1992, Numerical Recipes in Fortran, second edition (USA: Cambridge University Press)

Reich, W. 1982, A\&AS, 48, 219

Reich, P., \& Reich, W. 1986, A\&AS, 63, 205

Reich, P., \& Reich, W. 1988, A\&AS, 74, 7

Schlegel, D. J., Finkbeiner, D. P., \& Davis, M. 1998, ApJ, 500, 525 